\title{
Malaria Vaccine Development: A Challenge for Africa
}

\author{
Musa Sunday Akagwu Unyene ${ }^{1,2}$ and Dr. Mrs. Ani Agatha (PhD) ${ }^{1}$ \\ 1. Department of Medical Microbiology, University of Jos, P.M.B 2084 Jos, Postal Code: 930001, Plateau \\ State, Nigeria \\ 2. ACET Nigeria Head Office, Flat 3, Floor 2, FCS National Secretariat Building, Opposite Building Materials \\ Market, Yakubu Gowon Way, P.O. Box 1347, Jos, Postal Code: 930001, Plateau State, Nigeria
}

\begin{abstract}
Malaria remains one of the most widespread and deadly tropical diseases with more than 300 million cases and more than one million deaths each year. Since ninety percent of the cases occur among children in Sub-Saharan Africa and the poorer communities are worst affected, malaria clearly posses a huge threat to the existence and development of Africa. A malaria vaccine could greatly reduce the effects of the disease in terms of suffering and loss of lives. It also could prevent the spread of malaria more cost effectively than any existing treatment. This review was undertaken to highlight the basic challenges of malaria vaccine development in Africa and specifically highlights and discusses: reported progress on malaria vaccine development across the globe and particularly in Africa; challenges associated with the success rate of malaria vaccine development efforts; and Africa-specific operational and resource challenges in malaria vaccine development.
\end{abstract}

\section{Introduction On Malaria Vaccine Development}

Malaria has been one of the most deadly human infectious diseases in the world, and it is still the cause of about 2 million deaths per year, mainly among young children (under 5 years) and pregnant women (17). Each year, close to five billion episodes of clinical malaria occur worldwide, with Africa having more than $90 \%$ of this burden. Almost 3\% of disability adjusted life years are due to malaria mortality globally, $10 \%$ in Africa (8). One African child dies from malaria every 30 seconds (50). 109 countries were endemic for malaria in 2008, 45 of which fall within the World Health Organization (WHO) African region (51).

It is obvious that implementation of malaria control strategies such as insecticide-treated bednets and chemotherapy has reduced malaria related mortality substantially. However, it is needful to consider the use of an effective malaria vaccine (35) as both malarial parasites and insect vectors are increasingly becoming resistant to anti-malarial agents and insecticides respectively (13). A safe and effective vaccine is essential to achieve significant and sustained reductions in malaria-related morbidity and mortality (43). But the development of a safe and efficacious malaria vaccine has remained an elusive goal for the past 80 years (25).

The task of developing a vaccine that is of potentially preventative benefit for malaria is a complex process. Malaria is caused by protozoan parasites of the genus Plasmodium and transmitted by female Anopheles mosquitoes. Four species of Plasmodium are infectious for humans: Plasmodium falciparum (the most deadly species, responsible for $80 \%$ of infections worldwide and $90 \%$ of malaria-related deaths), Plasmodium vivax, Plasmodium malariae, and Plasmodium ovale (17). By their very nature, parasites are more complex organisms than bacteria and viruses, with more complicated structures and life-cycles (23). Plasmodium has a multi-stage life cycle, during which the parasite presents multiple antigens that show significant variability (25). In addition to the complexity of the parasite, is the fact that the populations bearing the burden of malaria also live in economically poor part of the world. Development of anti-malarial vaccines is therefore, a very tough challenge from technical, delivery and affordability points of view (14).

Scientists are however, optimistic about getting an effective malaria vaccine in the nearest future. Recent years have witnessed a new impetus and the generation of conclusive evidence that effective immunity against Plasmodium falciparum infection and severe disease can be induced through vaccination in children living in malaria-endemic areas. It is likely that the next decade will witness the first registration of a malaria vaccine. This vaccine is unlikely to provide complete protection, but it may become a useful tool to be added to the armoury in the fight against malaria (25).

At present there are approximately 100 candidate malaria vaccines in development, including preerythrocytic stage vaccines, blood-stage vaccines, anti-toxic vaccines, and transmission-blocking vaccines. Nevertheless, most of them are based on a small number of $P$. falciparum antigens $(50,40)$. Only a few candidates have entered clinical phase studies, and most are still at the pre-clinical phases (25). 


\section{Progress In Malaria Vaccine Development}

\section{History of Malaria Vaccines}

The parasite causing malaria, Plasmodium, was first identified by Charles Louis Alphonse Laveran, a French army surgeon stationed in Constantine, Algeria in 1880 (11, 23). Camillo Golgi, an Italian neurophysiologist, established in 1886 that there were at least two forms of malaria. In 1899, transmission of the human Plasmodium spp by mosquitoes and the complete sporogonic cycle of Plasmodium falciparum, $P$. vivax, and $P$. malariae were demonstrated by a team of Italian investigators led by Giovanni Batista Grassi, which included Amico Bignami and Giuseppe Bastianelli (11).

In 1955, a book entitled Man's Mastery of Malaria reflected the generally held views of that time. It is now half a century from then and malaria still kills 2-3 million people a year. Most of these deaths are in children younger than 5 years living in sub-Saharan Africa; one African child dies from malaria every 30 seconds. Rarely has scientific optimism been so misplaced (35). According to WHO reports, in 2004, P. falciparum was considered one of the leading causes of death worldwide, induced by a single infectious agent (13). In 2006, there were approximately 247 million malaria cases among 3.3 billion people at risk, causing nearly a million deaths. One hundred and nine countries were endemic for malaria in 2008, 45 of which fall within the WHO African region (52).

GlaxoSmithKline Biologicals (GSK) embarked on extensive research in the academic sector beginning in the 1960s, which revealed that hyperimmunization with radiation-attenuated sporozoites could protect rodents, primates, and ultimately human volunteers from malaria infection. The circumsporozoite protein (CSP), a sporozoite surface antigen, was identified as the likely target of the protective immune responses, and the gene encoding the CSP of Plasmodium falciparum was cloned and sequenced by scientists at the US National Institutes for Health and the Walter Reed Army Institute of Research (WRAIR), thereby ushering in the era of subunit vaccine development for malaria (6).

1973 saw the first report of human protection from malaria by vaccination. However, the vaccination consisted of the bites of about a thousand mosquitoes infected with malaria parasites that had been X-irradiated. This demonstration was obviously unlikely to be a practical means of mass vaccination. Much speculation and excitement was generated by the Spf66 candidate vaccine, despite uncertainty about how such a construct could work. Eventually, phase III trials showed that Spf66 lacked efficacy (35).

In a Belgian laboratory in 1987, a team of scientists fused a genetically engineered version of the evasive circumsporozoite protein - RTS,S that gives malaria its deadly edge on the human immune system to a surface antigen molecule. Not only was RTS,S the first vaccine capable of taking on a parasite, its invention was also unprecedented for focusing the intellectual and financial might of the pharmaceutical industry on cashstrapped communities across sub-Saharan Africa, where malaria kills nearly a million children each year (3).

It was reported on 29th July 2010 that European researchers, in what is being touted as a major breakthrough for malaria treatment, have shown that infected mice that are administered antibiotics (clindamycin and azithromycin) develop immunity against becoming infected again. There is currently no safe, effective vaccine against malaria (42), but this new, needle-free discovery could allow for a "naturally occurring transmission of the 'vaccine dose' by mosquito bites," Dr. Steffen Borrman, of the Heidelberg University School of Medicine and the head of research for the study, told LifeScientist.com.au (18).

\section{Approaches to Malaria Vaccine Development}

Malaria vaccines are designed to target certain stages of the malaria parasite's life cycle. The Plasmodium spp. has a complex life cycle consisting of distinct phases (7), such as asexual (pre-erythrocytic and erythrocytic in humans), and sexual (in mosquito). The life cycle starts when a female mosquito, while feeding on the blood of a healthy individual, injects sporozoites of Plasmodium into the body (24).

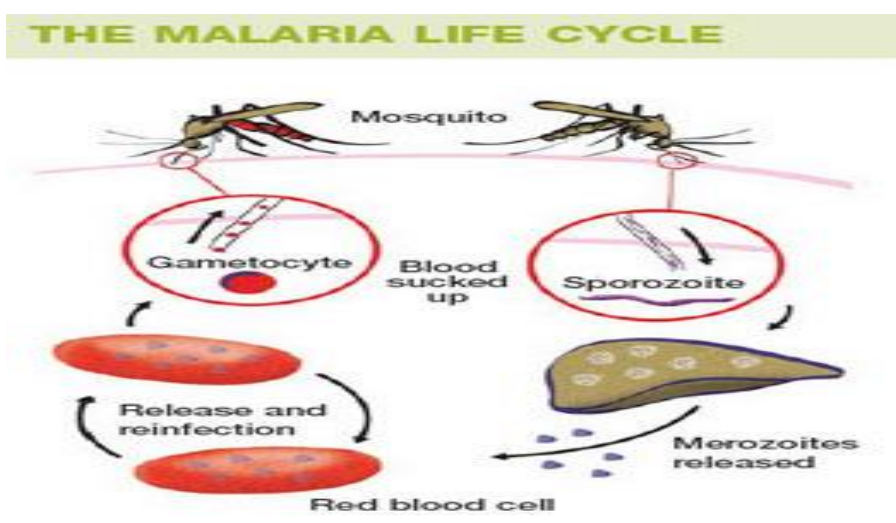


In order to develop malaria, an interaction should occur between three populations: humans, Anopheles (vector) and the parasite. The parasite adapts through mitotic mutations and meiotic recombination to each one of these different hosts (31).

Although the complex life cycle of Plasmodium is challenging for anti-malarial vaccine development, it offers many antigen targets as opportunities for combating malaria. For example, the existence of the multistage life cycle parasite allows scientists to intervene at different stages of the parasite's life cycle. The malaria spreading can also be stopped or significantly reduced by interrupting the normal life cycle of the parasite, including the period when the parasite is found in its vector, mosquito (14).

During the past decade, many candidate vaccine approaches have been tested in clinical trials. Many potential candidate vaccines now warrant preclinical assessment (35). Presently, there is a huge variety of vaccine candidates on the table. Pre-erythrocytic vaccines (which target the parasite before it reaches the blood), in particular vaccines based on CSP, make up the largest group. Other vaccine candidates include: those that seek to induce immunity to the blood stages of the infection; those that seek to avoid more severe pathologies of malaria by preventing adherence of the parasite to blood venules and placenta; and transmission-blocking vaccines that would stop the development of the parasite in the mosquito right after the mosquito has taken a blood-meal from an infected person (33). Antigen targets for potential candidate vaccines are highlighted as follows (14):

\section{Vaccines targeting pre-erythrocytic asexual stage antigens}

The malaria vaccine candidates against pre-erythrocyte stages include circumsporozoite protein (CSP) positioned intra-vascularly, the liver stage antigens (LSA-1 and LSA-3) which are intra-hepatocytic, and sporozoite surface protein-2 (SSP2)/Thrombospondin-related anonymous protein (TRAP), and CSP and TRAPrelated protein (CTRP). Pre-erythrocytic vaccines play an important role in protecting against malaria infection and provide, in best cases, sterilising humoral immunity by eliciting antibodies that target the invading sporozoites and preventing them from invading the liver (24).

\section{Vaccines targeting erythrocytic asexual blood stream antigens}

Malaria vaccine candidates against erythrocyte stages include an asexual blood stream stage developing in humans: merozoite surface proteins (MSP-1, MSP-2, MSP-3, MSP-4); apical membrane antigen 1 (AMA-1); erythrocyte binding antigen 175 (EBA-175); RAP1/2, Pf 35). Topolska, Lidgett, Truman, Fujioka and Coppel (2003) considered 10 different merozoite surface proteins, all present in Plasmodium falciparum. However, these MSPs are not all present in all Plasmodium spp., as there are examples such as MSP-2 that is not present in Plasmodium vivax.

\section{Vaccines targeting transmission-blocking candidate antigens}

Transmission blocking vaccines (TBV) are erythrocytic sexual blood stream vaccine candidate antigens. TBV can be divided into two target antigens: pre-fertilization antigens (including Pfs $48 / 45$ and Pfs 230 antigens found at the surface of the male and female gametes of malaria parasites) and the other postfertilization antigens (including Pfs 25 and Pfs 28). The major advantages of post-fertilization target antigens are the strong immunogenicity and the limited pre-existing antigenic polymorphism (47). Surface proteins expressed on the zygotes and mature ookinetes of the malaria parasites represent the second class of malaria transmission (10). According to Coban et al. (2004), antibodies directed against Pfs 25 completely block pathogen transmission (14). Expressed on the surface of late ookinetes, Pfs 28 is involved in adherence to the mosquito's gut epithelium (27).

\section{Multi-stage multi-epitope/antigen combination vaccines}

Most of the candidate vaccines are based on different technological platforms such as recombinant proteins, synthetic peptides, viral vectors, bacterial vectors, plasmid DNA and attenuated organisms (Doolan \& Stewart, 2007). Most of the vaccines under clinical trial are based on single antigen from a particular stage of Plasmodium life cycle. To effectively control the spread of malaria, it will be essential to create a multi-stagespecific complex vaccine or several individual stage-specific subunit vaccines singly or in combination using most non-polymorphic region(s) of the promising candidate antigens.

Several vaccines are developed recently based on the above idea including PfCP-2.9, a vaccine candidate consisting of the C-terminal regions of two leading malaria vaccine candidates - domain III of apical membrane antigen-1 (AMA-1) and 19-kDa C-terminal fragment of the MSP-1. Both of these regions are least polymorphic portions of AMA-1 and MSP-1, respectively; therefore most conservative antigens for combination vaccine candidate. The two candidates (AMA-1 and MSP-1) enhance the product yield and immunogenicity of the individual components when combined. The protective immune responses induced by either AMA-1 or MSP1-19 are dependent on protein conformation (38). 
In general, when two antigens are expressed together in a combination vector there may be antigenic competition. Fortunately, this vaccine candidate induces antibodies that inhibit blood-stage parasite growth in vitro and results showed no evidence of antigen competition. However, it revealed that sera from animals immunized with combination antigens recognised both the blood-stage parasite and the sporozoite. PfCP-2.9 chimeric protein previously appeared to be highly immunogenic in rabbits and monkeys. As the anti-AMA1(III) antibody titre induced by PfCP- 2.9 was 18 -fold higher than that induced by AMA-1(III) and the antiMSP1-19 antibody titre was 11-fold higher than that induced by MSP1-19 (38), it was suggested that PfCP-2.9 in combination with these recombinant pre-erythrocytic antigens induced antibodies that inhibited growth of blood-stage malarial parasites. Responding to its potential, promise and prospect the Chinese government gave its largest ever biopharmaceutical grant to Sinobiomed for development of PfCP2.9 vaccine (14).

Research and development into whole organism malaria vaccines is progressing rapidly with discovery of potential new candidate vaccines and the start of the first phase 1/2a clinical trial of the radiation attenuated sporozoite approach for Plasmodium falciparum. A group of leading scientists, clinical trialists and stakeholders, together with representatives of regulatory authorities including some from African countries, met in 2010 to document the issues that will require detailed consideration to assess this promising approach. Questions related to scale-up, quality, purity and consistency of a manufacturing process using mosquitoes to generate a commercial product, and demonstration of the stability of attenuated sporozoites will need further work (39).

\section{Malaria Vaccine Trials}

1973 saw the first report of human protection from malaria by vaccination. For about 20 years, progress occurred mainly in experimental models rather than in human vaccine trials (35). After four decades of research effort to develop a malaria vaccine, until now although more than 80 malaria candidate vaccines have undergone preclinical trial of which approximately 30 undergone clinical trials, only two of them underwent phase $2 \mathrm{~b}$ trial (14).

The first vaccine developed that has undergone field trials, is the SPf66, developed by Manuel Elkin Patarroyo in 1987. It presents a combination of antigens from the sporozoite (using CS repeats) and merozoite parasites. During phase I trials a $75 \%$ efficacy rate was demonstrated and the vaccine appeared to be immunogenic and well tolerated by subjects. The phase $2 \mathrm{~b}$ and 3 trials were less promising, with the efficacy falling to between $38.8 \%$ and $60.2 \%$. As gathered from Wikipedia, 2010 a trial was carried out in Tanzania in 1993 demonstrating the efficacy to be $31 \%$ after a year's follow up, however the most recent (though controversial) study in The Gambia did not show any effect. Despite the relatively long trial periods and the number of studies carried out, it is still not known how the SPf66 vaccine confers immunity; it therefore remains an unlikely solution to malaria (35). The efficacy of Patarroyo's vaccine has been disputed with some US scientists concluding in The Lancet (1997) that "the vaccine was not effective and should be dropped" while the Colombian accused them of "arrogance" putting down their assertions to the fact that he came from a developing country.

The CSP was the next vaccine developed that initially appeared promising enough to undergo trials. It is also based on the circumsporoziote protein, but additionally has the recombinant (Asn-Ala-Pro15Asn-ValAsp-Pro)2-Leu-Arg(R32LR) protein covalently bound to a purified Pseudomonas aeruginosa toxin (A9). However at an early stage a complete lack of protective immunity was demonstrated in those inoculated. The study group used in Kenya had an $82 \%$ incidence of parasitaemia whilst the control group only had an $89 \%$ incidence. The vaccine intended to cause an increased T-lymphocyte response in those exposed.

The RTS,S/AS02A vaccine is the candidate furthermost in vaccine trials. It is being developed by a partnership between the PATH Malaria Vaccine Initiative (a grantee of the Gates Foundation), the pharmaceutical company, GlaxoSmithKline, and the Walter Reed Army Institute of Research (26). In the vaccine, a portion of CSP has been fused to the immunogenic "S antigen" of the hepatitis B virus; this recombinant protein is injected alongside the potent AS02A adjuvant (33). In October 2004, the RTS,S/AS02A researchers announced results of a Phase IIb trial, indicating the vaccine reduced infection risk by approximately $30 \%$ and severity of infection by over 50\%. The study looked at over 2,000 Mozambican children (2). More recent testing of the RTS,S/AS02A vaccine has focused on the safety and efficacy of administering it earlier in infancy: In October 2007, the researchers announced results of a phase I/IIb trial conducted on 214 Mozambican infants between the ages of 10 and 18 months in which the full three-dose course of the vaccine led to a $62 \%$ reduction of infection with no serious side-effects save some pain at the point of injection (5). But research work on this vaccine is not complete for release yet.

\section{Malaria Vaccine Development Challenges}

An effective vaccine is an urgently needed intervention (45). Why, then, when there are vaccines against many infectious agents, do we still lack an effective vaccine against malaria? Professor Kevin Marsh, 
Director of the KEMRI-Wellcome Collaborative Research Programme in Kenya says: "Vaccination is historically the most effective way to control infectious disease but when you talk about vaccines, people usually think about those against bacteria and viruses. Malaria is a protozoal parasite, bigger and considerably more complex than viruses. Developing a vaccine against malaria is, therefore, more similar in concept to developing one against cancer than measles" (23).

Technical challenges of malaria vaccine development include identification of epitopes/antigens against appropriate targets, construction of DNA vector(s) that will express properly folded functional protein. Vaccine delivery challenges include developing an easy method to deliver multiple doses within a short period of time to infants and children of less than five years of age (14). Trials challenges include mobilising local experts, upgrading facilities, winning over government and convincing them of the importance of malaria vaccine and cultural acceptability among the low literate populations where the malaria burden is most pressing (3). Affordability challenges include development of cost-effective vaccines that can be stored at room temperature and be easily delivered (14).

\section{Immunological Challenges}

Immunity to malaria does occur naturally, but only in response to repeated infection with multiple strains of malaria (19). The first promising studies demonstrating the potential for a malaria vaccine were performed in 1967 by immunizing mice with live, radiation-attenuated sporozoites, providing protection to about $60 \%$ of the mice upon subsequent injection with normal, viable sporozoites $(36,14)$. Since the 1970s, there has been a considerable effort to develop similar vaccination strategies within humans. It was determined that an individual can be protected from a P. falciparum infection if they receive over 1,000 bites from infected, irradiated mosquitoes (28).

The Plasmodium is a highly complex parasite, with a multi-stage life cycle, during which the parasite presents multiple antigens that show significant variability. The main hindrance to developing a malaria vaccine is the choice of the antigen(s) to be included. This process has often relied on the use of imperfect animal models, in vitro assays with significant limitations, and sero-epidemiological assessments of naturally acquired immune responses in humans and their relationship to the risk of malaria infection or disease. However, we still have no surrogate measure of protection for malaria. Numerous associations between antigen-specific immune responses and the risk of infection or disease have been described. However, an association between a given immune response and the risk of malaria does not mean that those responses play a role in protection, as previous exposure to the parasite and development of responses to multiple antigens, most of which are unlikely to be linked to protection and are simply a marker of exposure, become an important confounding factor (2).

The complex life cycle of Plasmodium is challenging for anti-malarial vaccine development. It is believed that the immune mechanisms responsible for protection against malaria will require a multiple of 10-15 antigen targets for proper protection against various stages of malarial infection. Moreover, each antigen is reported to be highly polymorphic in nature (14). The various stages of the malaria parasite lifecycle have differing protective immune mechanisms and clinical endpoints, and usually different, often polymorphic, antigens (45). This is evident in the review of some existing vaccine candidates highlighted below.

\section{Vaccines targeting pre-erythrocytic asexual stage antigens}

The CSP is a 58-kDa protein and denominates the major component of the surface of the sporozoite (12). Before the initiation of the malaria infection, the mosquito injects the parasite into the vertebrate host. The CSP antigen is vital in protective immunity against the pre-erythrocytic stages of the Plasmodium infection (30). CSP has been identified as playing a crucial role in the invasion of hepatocytes by sporozoites (22).

TRAP is an essential protein for sporozoite invasion (48). The TRAP protein can be found within the micronemes of the mosquito sporozoite stage. TRAP-deficient sporozoites are unable to invade mosquito salivary gland cells or liver cells, due to lack of gliding ability. It is considered that the cytoplasmic domain of TRAP is involved in connecting the parasite actin-myosin machinery with the external substrate (22).

CTRP is essential for the Plasmodium falciparum's development in the mosquito's midgut. It also has an important role in the recognition and motility processes at the ookinete cell surface. When Anophles mosquitoes were fed with disrupted CTRP lines, oocyst formation was not observed despite the development of mature ookinetes (44).

\section{Vaccines targeting erythrocytic asexual blood stream antigens}

MSP-1 is a $195-\mathrm{kDa}$ protein. Research on MSP-1 has proven that it can induce a protective immune response against infections caused by the malaria parasite (49). MSP-1 presents high antigen diversity. However, its polymorphism might become an obstacle for effective vaccines. The intragenic recombination between MSP-1 alleles is a major mechanism for the generation of allelic variation in MSP-1. This 
recombination occurs in the meiotic phase, in the vector mosquito gut, right after receiving blood containing male and female gametocytes, the frequency with which recombination occurs being highly important (41).

Apical Membrane Antigen-1 (AMA-1) may also be considered as a sequence of the multisubunit vaccine for both $P$. falciparum and Plasmodium vivax, represented by an integral protein of $83 \mathrm{kDa}$ with 16 cysteine conserved residues. AMA-1 vaccine has been proven safe and capable of inducing immunity in European adults; studies conducted in Mali in 2007 by African Malaria Network Trust also confirmed its safety on African healthy population. Studies have shown that antibodies directed against AMA-1 neutralise the invasion of merozoites. Highly polymorphic with most of polymorphisms occurring in domain I, AMA-1 could hardly create a broadly effective vaccine. Its recombination, however, can induce protective immune responses in mouse (34), and monkey models of malaria. Domain III of AMA-1, which is less polymorphic or nonpolymorphic, constitutes the potential vaccine candidate, its monoclonal and polyclonal antibodies inhibiting the merozoite invasion of erythrocytes. Research conducted in the Wosera region of Papua New Guinea and focused on the AMA-1 diversity in a large number of symptomatic and asymptomatic $P$. falciparum infections brought new information of the malaria vaccine candidate's diversity in a single population. Various results indicated that, polymorphisms in AMA-1 domain I are the result of selective pressure exerted by protective immune responses (16).

EBA-175 also induces antibodies that inhibit malaria merozoite invasion. Mice cultivated antibodies against EBA-peptide 4 block binding of native EBA-175 to human erythrocytes and inhibit merozoite invasion in vitro. Biological and parasitological studies performed by Fousseyni, Toure, Deloron and Migot-Nabias (2006) on 158 children from a village in southeast Gabon during the whole malaria transmission season of 1995 allowed the researchers to distinguish between protected and unprotected children. The study revealed that IgG antibodies competed for binding to EBA-175 epitopes and was consistent with the role of an isotype imbalance in the resistance and/or susceptibility to malaria infection. A different study conducted in three locations in Zimbabwe revealed that antibody levels against malaria antigens are distinct with regard to altitude and climatic conditions. Analysis revealed an age associated statistical significant pattern of reactivity to EBA-175, this finding is consistent with that of Okenu et al. (2000) and may suggest that antibodies to EBA- 175 may represent a marker of age acquired immunity. Antibodies that bind to EBA-175 may interfere with the interaction of EBA-175 with sialic acid residues of glycophorin A on erythrocytes, a crucial process in the sialic acid-dependent parasite invasion pathway (34).

\section{Vaccines targeting transmission-blocking candidate antigens}

Pfs $48 / 45$ and Pfs 230 are pre-fertilization antigens found at the surface of the male and female gametes of malaria parasites. Pfs $48 / 45$ contains three of the cysteine domains responsible for male gamete fertility, while Pfs 230 contains fourteen of the cysteine domains (47). Monoclonal antibodies against these molecules can impede the transmission of parasites to mosquitoes (10).

Pfs 25 and Pfs 28 are post-fertilization antigens. The major advantages of post-fertilization target antigens are the strong immunogenicity and the limited pre-existing antigenic polymorphism (47). Surface proteins expressed on the zygotes and mature ookinetes of the malaria parasites represent the second class of malaria transmission (10). The Pfs 25 and Pfs 28 proteins are rich in cysteines, but their arrangements are different compared to the first class proteins. They correspond to a series of four complete (six cysteines) or partial (four cysteines) EGF-like domains (10). According to Coban et al. (2004), antibodies directed against Pfs 25 completely block pathogen transmission. Pfs 25 material is being used for generating immune sera in mice and monkeys. Pfs 28 is a $28-\mathrm{kDa}$ protein, supposedly anchored to parasite surface by glycosylphosphatidylinositol, encoded on chromosome 10 and sharing putative functional determinants with Pfs25. Expressed on the surface of late ookinetes, Pfs 28 is involved in adherence to the mosquito's gut epithelium (27).

\section{Multi-stage multi-epitope/antigen combination vaccines}

PfCP-2.9 is a vaccine candidate consisting of the C-terminal regions of two leading malaria vaccine candidates, domain III of apical membrane antigen-1 (AMA-1) and 19-kDa C-terminal fragment of the MSP-1. Both of these regions are least polymorphic portions of AMA-1 and MSP-1, respectively therefore most conservative antigens for combination vaccine candidate. It contains 18 cysteine residues, six in AMA-1(III) and twelve in MSP1-19, to form nine intra-molecular disulfide bonds (38).

\section{Programmatic and Operational Challenges for Africa Systems Challenges}

Although the development of malaria vaccines is a priority for Africa, until recently, "the process of antigen discovery and validation is mainly restricted to universities and public research institutions in the north (Europe). Industry is generally uninterested in malaria vaccine development. Malaria vaccine trials in Africa favour sites with historical affiliations to Europe (UK and France); recently the USA entered the race. Trials at 
neutral or African led or African owned sites are rare, although they are essential for multi-centre Phase III trials" (29).

Research and development into whole organism malaria vaccines is progressing rapidly including the discovery of potential new candidate vaccines and the start of the first phase 1/2a clinical trial of the radiation attenuated sporozoite approach for Plasmodium falciparum. Questions related to scale-up, quality, purity and consistency of a manufacturing process using mosquitoes to generate a commercial product, and demonstration of the stability of attenuated sporozoites will need further work. Should a high level of efficacy be demonstrated in clinical challenge studies, it will become a priority to agree in which populations and age groups questions about strain-transcendence and duration of efficacy should be answered, and how clinical development can progress with an approach based on cryopreservation in liquid nitrogen (39).

Insufficient clinical trial capacity and normative research functions such as local ethical committee reviews contribute to slow down malaria vaccine development process (24). Africa led and/or owned institutions and networks concerned with malaria vaccine research and development such as the African Malaria Network Trust (AMANET) are needed in good number with offices in all endemic nations of Africa. AMANET whose mission is to promote capacity strengthening of African malaria research institutions, was founded in 2002 and is currently focusing on malaria vaccine development. AMANET has trained over 900 African malaria researchers at workshops relevant to clinical trials of candidate malaria vaccines that will meet scientific, ethical, and international Good Clinical Practice standards (9).

\section{Cultural Challenges of Malaria Vaccine Research in Africa}

Joe Cohen, one of the inventors of the RTS,S malaria vaccine, who still works on vaccines for emerging diseases and HIV/AIDS with GlaxoSmithKline said, "Whenever you start a malaria trial you have to have a strong heart. No matter how much planning in advance and looking at transmission data years pretrial, you really never know what's going to happen during the year you are doing the trial."

Scientists running the world's largest malaria vaccine trial face the challenge of winning the support of local communities in sub-Saharan Africa to do their research. Reports from the RTS,S trial sites in Kilifi, Kenya reflect the following challenges (3):

The RTS,S is the first vaccine capable of taking on a parasite, its invention was also unprecedented for focusing the intellectual and financial might of the pharmaceutical industry on cash-strapped communities across sub-Saharan Africa, where malaria kills nearly a million children each year. But the vaccine's creators could hardly have known how the pristine logarithms of the laboratory would play out on a continent of floods, scarce infrastructure, and threadbare health systems.

The challenges were immense - hundreds of millions of dollars had to be raised to finance a vaccine with no prospect for commercial success given the impoverished target population. A network of local experts had to be set up and supplied with world class facilities, and an array of often fractious and at times less-thanethical governments had to be won over and convinced of the importance of a vaccine meant to help the most marginalised of their people.

Over 22 years after the RTS,S was engineered, with money from the Bill \& Melinda Gates Foundation and a chain of research collaborations stretching across 11 trial sites in seven countries, the largest and most advanced trial ever of a malaria vaccine began in May 2009.

Approval of the RTS,S will come down to much more than its biological viability. The success of the vaccine relies on the communities whom the RTS,S could benefit the most - people from places that could not be more removed from the sterile chambers of western science. People like Timothy Mwadondo, who was born in Junju, a tiny village in Kenya known for its coconut moonshine, in the same year RTS,S was invented. What happened to Mwadondo illuminates the challenges that trial organisers will have in winning over the population.

Across much of sub-Saharan Africa communities confront an epidemiological minefield without the benefits of basic health or education. They blame demons and witchcraft when children have fevers brought on by pneumonia, violent wasting from stomach parasites, or seizures that can come with severe malaria. In Junju, some whispered that researchers used blood samples from Junju's children to feed these demons (3).

Mwadondo was among dozens of field workers hired by scientists to monitor a few dozen participants in an earlier trial of RTS,S around Junju. Late one night, Mwadondo was called to visit a sick child. As he returned home, a group of men snatched him from the dark footpath, stole his clothing, and slipped a noose around his neck, before dragging him miles into the forest and abandoning him there. "It was a study participant's parent who did it", Mwadondo said. "They said I brought people who take the blood from their children to the devils, and that is what contributed to what happened" (3).

Health experts say such fears are bred by decades of neglect in countries with health systems corroded by poverty and government indifference. Too little comes too late and parents begin to associate health care with death. "They're not sure of all of the early danger signs", said Graham Reid of the International Development Research Centre (IDRC) in 2009. "If this kid goes into a coma in the facility, the mother is in the 
wrong place at the wrong time. This is not a condition that they can deal with, so she runs out and urinates on the child or does some traditional healing."

Poor governance does nothing to improve the situation. Even in Kenya, where standards are relatively high, scandal is commonplace. Days after chiefs in Kilifi called upon people to trust researchers carrying out the latest trial, the board of the Kenya Medical Research Institute which was a partner on the study, was sacked because it was unable to account for the disappearance of a staff pension fund worth millions of dollars.

It is not only for lack of a vaccine that malaria, a disease that has all but disappeared in the developed world, continues to kill nearly a million children yearly in sub-Saharan Africa, where poverty stunts health-care systems. "The people who are dying of malaria are the poor", said Justa Wawira (2009), spokesperson KEMRI/Wellcome Trust Programme in Kilifi. "It's not the rich. They can afford drugs; they can afford a good lifestyle with sprays and environmental hygiene."

The vast resource gap that has made such things an anomaly across much of southern Africa also poses a dilemma for anyone hoping to carry out state of the art vaccine trials on a grand scale here.

\section{Resource Challenges for Africa}

"African scientists have a key role to play, in their collaboration with their colleagues from the north (Europe) and, most importantly, by South-South collaboration."

According to Breman et.al, 2004 prominent African malaria researchers including Ntoumi affirm that the goals of malaria control may never be achieved without strong involvement of those scientists who are directly affected by this terrible disease in their daily life and until recently, true participation of African scientists in the international effort to control malaria has been minimal. The importance of networks to promote connection between African scientists is underscored, and inadequate dialogue between malaria researchers and control managers is lamented.

In the past, funding of malaria research was a major stumbling block towards faster progress in this area. MVI (2002) lamented, "Despite malaria's tremendous social and economic impact, global spending for malaria vaccine research and development $(R \& D)$ is far less than the estimated $\$ 300$ to $\$ 500$ million required to advance one vaccine through the product development process." Fortunately, now with the Gate's Foundation's GCE funding and funding from China, there is a hope that progress will be fast and an affordable malaria vaccine will be available in the near future (14).

\section{Discussion}

Malaria remains one of the most widespread and deadly tropical diseases with more than 300 million cases and more than one million deaths each year. Since ninety percent of the cases occur among children in Sub-Saharan Africa and the poorer communities are worst affected, malaria clearly posses a huge threat to the existence and development of Africa.

Research has shown that without a vaccine, malaria eradication has been largely achieved in developed countries through hygiene, effective drugs, and the reduction of mosquito breeding grounds via wetlands clearing, chemical treatment to control mosquito populations, and water-system management. These approaches have however, not been as effective in Africa and other developing countries for many reasons, including costs as well as the challenge of managing potential environmental and health effects of chemical parasite removal. In addition to these eradication challenges is the exacerbating problem of the malaria parasite and its insect vectors becoming resistant to existing drug treatments and therapeutic-control measures.

A malaria vaccine could greatly reduce the effects of the disease in terms of suffering and loss of lives. It also could prevent the spread of malaria more cost effectively than any existing treatment. Vaccine use would reduce the need for expensive, often unaffordable medicines and curb the problem of drug-resistant parasites. Moreover, vaccine use would reduce the need for chemical treatment to control mosquito populations, thus minimizing negative environmental effects. Preventive vaccines therefore present better hope for malaria eradication in Africa, and are sorely needed especially that it has been reported that various factors are now spreading malaria into areas previously free of infection.

Much work has been performed and over the past decade there has been considerable progress in the understanding of immune mechanisms involved in conferring protection to malaria and in the identification of vaccine candidate antigens and their genes. Several new vaccines have entered Phase I/II trials recently, new adjuvants have been developed for human use and new approaches, such as DNA vaccines and structural modification of antigens to circumvent some of the strategies the parasite uses to avoid the immune response, are being applied.

Based on this review, developing an effective malaria vaccine is possible but remains an enormous scientific and socioeconomic challenge, especially for Africa due to deficiency of required infrastructure necessary for vaccine research and Africa's commitment so far is not commensurate to their Western counterparts. Neglect for the poor, who actually are the worst affected populations in malaria endemic countries 
is a common African enigma. In order to develop an Africa-friendly malaria vaccine it is needful that basic infrastructural requirements for health care and research be made available and affordable for all.

The process of malaria vaccine development from pre-clinical research to licensing is complex and requires several years; it is possible however, that continuous scientific collaboration between African and Western scientists could motivate further interest in this area and especially in discovering a suitable surrogate of protection.

For an ideal malaria vaccine development, it is important to identify least polymorphic antigen(s) or epitope(s) that will induce maximum immunogenic response against majority of the parasites covering most diverse endemic areas. The lack of ideal antigen(s) or epitope(s) would however suggest the use of a multicomponent as suggested by Chowdhury et. al. (2009). To come by a safe, effective and affordable malaria vaccine, there is an urgent need for greater commitment and action of African scientists and governments towards the development of Africa-friendly malaria vaccine programmes.

The selection of new malaria vaccine trial sites should consider epidemiological, ownership and leadership criteria. Establishment of new trial sites should be guided by needs assessment process, followed by capacity strengthening to fill the gaps in personnel, equipment, transport and infrastructure. All systems (e.g. financial, management, ethics and regulatory review) should be strengthened. Given the enormity of the challenges involved, public-private partnerships are essential.

Government and non-governmental agencies working in malaria vaccines clinical trials should expedite action to bridge the human and infrastructural resource gaps across Africa. Malaria vaccines are essentially needful in order to curb the high morbidity and mortality rates from malaria. Global leaders must ensure that a more appropriate level of resources is applied to the challenge.

\section{Conclusion}

Successful development and use of a malaria vaccine will no doubt curb high morbidity and mortality rates caused by the disease. To achieve this, the combined efforts of in-country and global leaders with other interest groups is required for long term investment and support.

\section{References}

[1]. Agency for Science, Technology and Research (A*STAR), Singapore (2009). Effective Vaccine For Malaria Possible, Study Shows. ScienceDaily. Retrieved July 17, 2010, from http://www.sciencedaily.com /releases/2009/07/090730074340.htm

[2]. Alonso PL, Sacarlal J, Aponte JJ, Leach A, Macete E, Milman J, Mandomando I, Spiessens B, Guinovart C, Espasa M, Bassat Q, Aide P, Ofori-Anyinam O, Navia MM, Corachan S, Ceuppens M, Dubois MC, Demoitie MA, Dubovsky F, Menendez C, Tornieporth N, Ballou WR, Thompson R, Cohen J (2004). "Efficacy of the RTS,S/AS02A vaccine against Plasmodium falciparum infection and disease in young African children: randomised controlled trial". Lancet 364 (9443): 1411-20. doi:10.1016/S0140-6736(04)17223-1. PMID 15488216.

[3]. Alsop Z. (2009). Malaria vaccine researchers face cultural challenges. The Lancet, Volume 374, Issue 9684, Pages 104 - 105, 11 July 2009 doi:10.1016/S0140-6736(09)61269-1

[5]. Aponte, J. J., Aide, P., Renom, M., Mandomando, I., Bassat, Q., Sacarlal, J., Manaca, M. N., Lafuente, S., Barbosa, A., Leach, A., Lievens, M., Vekemans, J., Sigauque, B., Dubois, M., Demoitié, M., Sillman, M., Savarese, B., McNeil, J. G., Macete, E., Ballou, W. R., Cohen, J. and Alonso P. L. (2007). "Safety of the RTS,S/AS02D candidate malaria vaccine in infants living in a highly endemic area of Mozambique: a double blind randomised controlled phase I/IIb trial". Lancet 370 (9598): 1543-51. doi:10.1016/S0140-6736(07)61542-6. PMID 17949807.

[6]. Ballou, W. R., Cahill, C. P. (2007). Two Decades of Commitment to Malaria Vaccine Development: GlaxoSmithKline Biologicals. American Journal of Tropical Medicine and Hygiene, 77(6_Suppl), 2007, pp. 289-295 Copyright (C) 2007 by The American Society of Tropical Medicine and Hygiene

[7]. Boutin, J. P., Pradines, B., Pages, F., Legros, F., Rogier C., Migliani (2005). Epidemiology of malaria. Rev Prat. 55: 833-40.

[8]. Breman, J. G., Alilio, M. S., Mills, A. (2004). Conquering the Intolerable Burden of Malaria: What's New, What's Needed: A Summary. American Journal of Tropical Medicine and Hygiene, 71(2), pp. 1-15.The American Society of Tropical Medicine and Hygiene

[9]. Breman, J. G., Alilio, M. S., White, N. J. (2007). Towards an African-Driven Malaria Vaccine Development Program: History and Activities of the African Malaria Network Trust (AMANET). Defining and Defeating the Intolerable Burden of Malaria III: Progress and Perspectives: Supplement to Volume 77(6) of American Journal of Tropical Medicine and Hygiene

[10]. Carter, R. (2001). Transmission blocking malaria vaccines. Vaccine, 19:2309-2314.

[11]. CDC (2004). The History of Malaria, an Ancient Disease. April 23, 2004 Division of Parasitic Diseases National Center for Zoonotic, Vector-Borne, and Enteric Diseases (ZVED). Retrieved from http://www.cdc.gov/nczved/dpd

[12]. Chenet, S. M., Branch, L. H., Escalante, A. A., Lucas C. M., Bacon D. J. (2008). Genetic diversity of vaccine candidate antig ens in Plasmodium falciparum is isolates from the Amazon basin of Peru. Malaria Journal. 7:93, doi:10.21 86/14 75-28 75-7-93.

[13]. Chowdhury, K. and Bagasra O. (2007). An edible vaccine for malaria using transgenic tomatoes of varying sizes, shapes and colors to carry different antigens. Medical Hypotheses. 68(1):22-30.

[14]. Chowdhury, K., Kantor, M., Sestras, R. (2009). Malaria Vaccine Candidate Diversity Offers Challenges and Opportunities for Effective Vaccine Development. Notulae Botanicae Horti Agrobotanici Cluj-Napoca 37 (1) 2009, 9-16. ISSN 1842-4309 Retrieved from www.notulaebotanicae.ro

[15]. Coban, C., Ishii, K. J., Stowers, A. W., Keister, D. B., Klinman, D. M., Kumar, N. (2004). Effect of CpG Oligodeoxynucleotide s on the Immunogenicity of Pfs25, a Plasmodium faciparum Transmission-Blocking Vaccine Antigen. Infection and Immunity. 584-588. 
[16]. Cortes, A., Mellombo, M., Mueller, I., Benet, A., Reeder, J. C., Anders, R. F. (2003). Geographical Structure of Diversity and Differences between Symptomatic and Asymptomatic Infections for Plasmodium falciparum Vaccine Candidate AMA1. Infection and Immunity. 1416-1426.

[17]. Douradinha, B., Mota, M. M., Luty, A. J. F., Sauerwein, R. W. (2008). Cross-Species Immunity in Malaria Vaccine Development: Two, Three, or Even Four for the Price of One? Infection and Immunity, Mar. 2008, Vol. 76, No. 3 p. 873-878. 0019-9567/08/\$08.00_0 doi:10.1128/IAI.00431-07. American Society for Microbiology

[18]. Dulnier P. (2010). Breakthrough announced in search for malaria vaccine. VaccineNewsDaily.com on July 29, 2010. Retrieved from http://vaccinenewsdaily.com/on 09-08-2010

[19]. Färnert., A., Williams, T. N., Mwangi, T. W., Ehlin, A., Fegan, G., Macharia, A., Lowe, B. S., Montgomery, S. M., Marsh, K. (2009). "Transmission-dependent tolerance to multiclonal Plasmodium falciparum infection". J Infect Dis 200 (7): 1166-1175. doi:10.1086/605652. PMID 19702508.

[20]. Fousseyni, Toure, S, Deloron, P., Migot-Nabias (2006). Analysis of Human Antibodies to Erythrocyte Binding Antigen 175 peptide 4 of Plasmodium falciparum. Clinical Medicine and Research. 4(1): 1-6.

[21]. Gardner, M. J., Hall, N., Fung, E., White, O., Berriman, M., Hyman, R. W., Carlton, J. M., Pain, A., Nelson, K. E., Bowman, S., Paulsen, I. T., James, K., Eisen, J. A., Rutherford, K., Salzberg, S. L., Craig, A., Kyes, S., Chan, M., Nene, V., Shallom, S. J., Suh, B., Peterson, J., Angiuoli, S., Pertea, M., Allen, J., Selengut, J., Haft, D., Mather, M. W., Vaidya, A. B., Martin, D. M. A., Fairlamb, A. H., Fraunholz, M. J., Roos, D. S., Ralph, S. A., McFadden, G. I., Cummings, L. M., Subramanian, G. M., Mungall, C., Venter, J. C., Carucci, D. J., Hoffman, S. L., Newbold, C., Davis, R. W., Fraser C. M., Barrell, B. (2002). "Genome sequence of the human malaria parasite Plasmodium falciparum". Nature 370 (6906): 1543. doi:10.1038/nature01097. PMID 12368864.

[22]. Gilberger, T. W., Thompson, J. K., Reed, M. B., Good, R. T., Cowman A. F. (2003). The cytoplasmic domain of the Plasmodium falciparum ligand EBA-175 is essential for invasion but not protein trafficking. Journal of Cell Biology 162 (2): 317-327.

[23]. Giles, C. (2005). Why don't we have a malaria vaccine? Wellcome Trust and Malaria 27 Sept 2005. Retrieved from http://www.welcome.ac.uk/. on 09-08-2010

[24]. Girard, M. P., Reed, Z. H., Friede M., Kieny M. P. (2007). A review of human vaccine research and development. Malaria Vaccine. 25:1567-1580.

[25]. Guinovart, C., Alonso, P. L. (2007). Methods for Determining Vaccine Efficacy and Effectiveness and the Main Barriers to Developing a Fully Deployable Malaria Vaccine. American Journal of Tropical Medicine and Hygiene, 77(6_Suppl), 2007, pp. 276-281. Copyright () 2007 by The American Society of Tropical Medicine and Hygiene Retrieved from http://www.ajtmh.org/misc/terms.shtml on 17-07-2010

[26]. Heppner, D. G., Kester, K. E., Ockenhouse, C. F., Tornieporth, N., Ofori, O., Lyon, J. A., Stewart, V. A., Dubois, P., Lanar, D. E., Krzych, U., Moris, P., Angov, E., Cummings, J. F., Leach, A., Hall, B. T., Dutta, S., Schwenk, R., Hillier, C., Barbosa, A., Ware, L. A., Nair, L., Darko, C. A., Withers, M. R., Ogutu, B., Polhemus, M. E., Fukuda, M., Pichyangkul, S., Gettyacamin, M., Diggs, C., Soisson, L., Milman, J., Dubois, M., GarÅ§on, N., Tucker, K., Wittes, J., Plowe, C. V., Thera, M. A., Duombo, O. K., Pau, M. G., Goudsmit, J., Ballou, W. R., Cohen, J. (2005). "Towards an RTS,S-based, multi-stage, multiantigen vaccine against falciparum malaria: progress at the Walter Reed Army Institute of Research". Vaccine 23 (17-18): 224350. doi:10.1016/j.vaccine.2005.01.142. PMID 15755604.

[27]. Hisaeda, H., Stowers, A. W., Tsuboi, T., Collins W. E. (2000). Antibodies to Malaria vaccine candidates Pvs 25 and Pvs 28 completely block the ability of Plasmodium vivax to infect Mosquitoes. Infection and Immunity 6618-6623.

[28]. Hoffman, S. L., Goh, L. M. L, Luke, T. C., Schneider, I., Le, T. P., Doolan, D. L., Sacci, J., Vega, P., Dowler, M., Paul, C., Gordon, D. M., Stoute, J. A., Church, L. W. P., Sedegah, M., Heppner, D. G., Ballou, W. R., Richie, T. L. (2002). "Protection of humans against malaria by immunization with radiation-attenuated Plasmodium falciparum sporozoites". J. Infect. Dis. 185 (8): 1155-64. doi:10.1086/339409. PMID 11930326.

[29]. lama W. L. (2003). Malaria vaccines in Africa Acta Tropica Volume 88, Issue 2, October 2003, Pages 153-159. doi:10.1016/S0001-706X(03)00200-6. Copyright @ 2003 Elsevier B.V.

[30]. Kumkhaek, C., Phra-ek, K., Renia, L., Singhasivanon, P., Looareesuwan, S., Hirunpetcharat, C., White, N. J., Brockman, A., Gruner, A.C., Lebrun, N., Alloueche, A., Nosten, F., Khusmith, S., Snounou, G. (2005). Are Extensive T Cell Epitope Polymorphisms in the Plasmodium falciparum Circumsporozoite Antigen, A Leading Sporozoite Vaccine Candidate Selected by Immune Pressure? The Journal of Immunology. 175:3935-3939.

[31]. Le Bras, J., Durand R. (2003). The mechanisms of resistance to antimalarial drugs in Plasmodium falciparum. Fundam Clin Pharmacol. 17:147-153.

[32]. Malaria Vaccine Initiative (2002). Malaria Vaccine R\&D: The Case for Greater Resources

[33]. Matuschewski, K. (2006). "Vaccine development against malaria". Curr Opin Immunol 18 (4): $449-57$. doi:10.1016/j.coi.2006.05.004. PMID 16765576.

[34]. Mlambo, G., Mutambu, S.L., Mduluza, T., Soko, W., Mbedzi, J., Chivenga, J., Lanar, D.E, Singh, S., Carucci, D., Gemperli, A., Kumar, N. (2006). Antibody responses to Plasmodium falciparum vaccine candidate antigens in three areas distinct with respect to altitude. Acta Tropica. 100:70-78.

[35]. Moorthy, V. S., Good, M. F., Hill, A. V. S. (2004). Malaria vaccine developments. The Lancet Vol 363 January 10, 2004 www.thelancet.com

[36]. Nussenzweig, R., Vanderberg, J., Most, H., Orton, C. (1967). "Protective immunity produced by the injection of x-irradiated sporozoites of plasmodium berghei". Nature 216 (5111): 160-2. doi:10.1038/216160a0. PMID 6057225.

[37]. Okenu, D. M., Riley, E. M., Bickle, Q. D., Agomo, P. U., Barbosa A., Daugherty, J. R., Lanar, D.E., Conway, D. J. (2000). Analysis of human antibodies to erythrocyte binding antigen 175 of plasmodium falciparum. Infect immune. 68:5559-5566.

[38]. Pan, W., Huang, D., Zhang, Q., Qu, L., Zhang, D., Zhang, X., Xue X., Qian, F. (2004). Fusion of Two Malaria Vaccine Candidate Antigens Enhances Product Yeild, Immunogenicity, and Antibody - Mediated Inhibition of Parasite Growth In Vitro. The Journal of Immunology. 172:6167-6174

[39]. Pinder, M., Moorthy, V.S., Akanmori, B.D., Genton, B., Brown G.V. (2010). MALVAC 2009: Progress and Challenges in Development of Whole Organism Malaria Vaccines for Endemic Countries, 3-4 June 2009, Dakar, Senegal Vaccine, Volume 28, Issue 30, 5 July 2010, Pages 4695-4702

[40]. Reed, Z. H., Friede, M., Kieny, M. P. (2006). Malaria vaccine development: progress and challenges. Curr Mol Med 6: 231245.[Web of Science][Medline] 
[41]. Sakihamaa, N., Kanekob, A., Hattoric, T., Tanabea, K. (2001). Limited recombination events in merozoite surface protein-1 alleles of Plasmodium falciparum on Islands. Gene 279, 41-48.

[42]. Shotwell, S. L. (2007). Patent Consolidation and Equitable Access: PATH's Malaria Vaccines. In Intellectual Property Management in Health and Agricultural Innovation: A Handbook of Best Practices (eds. A Krattiger, RT Mahoney, L Nelsen, et al.). MIHR: Oxford, U.K., and PIPRA: Davis, U.S.A. Available online at www.ipHandbook.org.

[43]. Stevenson, M. M., Riley, E. M. (2010). Innate immunity to malaria; Nature Reviews Immunology 4, 169-180 (1 March 2004) doi:10.1038/nri1311. @ 2010 Nature Publishing Group, a division of Macmillan Publishers Limited. All Rights Reserved.

[44]. Templetion, T. J., Kaslow, D. C. Fidock, D. A. (2000). Developmental Arrest of the human malaria parasite Plasmodium falciparum within the mosquito midgut via CTRP gene disruption. Molecular Microbiology. 36 (1): 1-9.

[45]. Todryk, S. M., Hill, A. V. S. (2007). Malaria vaccines: the stage we are at; Nature Reviews Microbiology 5, 487-489 (1 July 2007) doi:10.1038/nrmicro1712

[46]. Topolska, A.E., Lidgett, A., Truman, D., Fujioka, H., Coppel, R. L. (2003). Characterisation of a membrane-associates rhoptry protein of Plasmidium falciparum. Journal Biol Chem, 279:4648-4656.

[47]. Tsuboi, T., Tachiband, M., Kaneko, O., Torrii M. (2003). Transmission-blocking vaccine of vivax malaria. Parasitology International. 52-11.World Health Organisation (2000). Malaria - a global crisis. Geneva: World Health Organization, 2000.

[48]. Weedall, G. D., Preston, B. M. J., Thomas, A. W., Sutherland, C. J., Conway, D. J. (2007). Differential evidence of natural selection on two leading sporozoite stae malaria vaccine candidate antigens. International Journal for Parasitology. 37:77-85.

[49]. Weiqing, P., Ravot, E., Tolle, R., Rainer, F., Mosbach, R., Turbachova, I., Bujard, H. (1999). Vaccine candidate MSP-1 from Plasmodium falciparum: a redesigned 4917 bp polynucleotide enables synthesis and isolation of full - length protein from Escherichia coli and mammalian cells. Nucleic Acids Research. 27 (4): 1094 - 1103 (10).

[50]. World Health Organization (2006). Candidate Malaria Vaccines in Pre-Clinical Development. Geneva: World Health Organization. Retrieved from http://www.who.int/vaccine_research/documents/RainbowTablePreclinical_December2006.pdf. on $17-07-2010$

[51]. World Health Organization (2008). World malaria report. 